\title{
Analysis of Degradation-compression-seepage Interaction in MSW Landfills by a BCHM Coupled Model
}

\author{
Hai-Long LIU ${ }^{1, a}$, Yu-Chao LI, b, ${ }^{\text {, }}$, Yun-Min CHEN ${ }^{1, c}$ \\ ${ }^{1}$ MOE Key Laboratory of Soft Soils and Geoenvironmental Engineering, Zhejiang University, \\ Hangzhou 310058, China \\ alhl.zju@live.com, ${ }^{b}$ liyuchao@zju.edu.cn, chenyunmin@zju.edu.cn \\ ${ }^{*}$ Corresponding author
}

Keywords: Landfill, Coupled Model, MSW with High Kitchen Waste Content.

\begin{abstract}
Municipal solid waste (MSW) is a special kind of porous media with a large amount of degradable composition. A biochemo-hydro-mechanical (BCHM) coupled model is presented with a consideration of MSW degradation characteristic different from traditional soils. The cellulose anaerobic degradation model is used to describe landfill gas and leachate generation during biodegradation. MSW deformation behavior and seepage characteristic are expressed by considering solid skeleton soften due to biodegradation. The seepage and settlement behaviors in a hypothetical landfill with high kitchen waste content are simulated by the presented model. The results demonstrate the problems in landfills with high kitchen waste content, such as leachate mound, leachate-gas movement mutual retardation, and significant closure settlement.
\end{abstract}

\section{Introduction}

Municipal solid waste (MSW) production increased significantly with the rapid development of urbanization of China. Landfilling is the main disposal method in China. According to report, there were 366 sanitary MSW landfills till 2007 which disposed more than $80 \%$ of MSW in China [1]. MSW is a special kind of porous media with a large amount of degradable composition, there are complicated biochemical reactions in landfills which would generate a large amount of leachate, gas and contamination. Landfill is not only a kind of earth structure, but also a large scale bioreactor. There are series of environmental problems due to improper management of landfills, such as: slope failure, leachate leakage and landfill gas emission even explosion.

The complicated biochemical process in landfills make engineering properties of MSW changing with time, which is the key different characteristic from traditional soil [2]. During the process of biochemical degradation, biodegradable components of MSW transform from the solid phase into the gas and liquid phase, which results pore volume increase and skeleton strength weaken. The weakened solid skeleton is compressed by overload, at the same time, the pore volume compression changed liquid and gas permeability of MSW. Leachate flow and gas transportation in landfill changed the pore pressure of water and gas, which reacted in the effective stress of MSW solid skeleton and made further compression.

Interactions between degradation, compression and seepage behaviors are very complex, and a research method which can take account in to these interactions is required to improve understanding of coupled mechanisms and management of landfills.

In recent years, many researchers began the numerical analysis of degradation, compression and seepage interactions in MSW, such as: Nastev et al. (2001) [3], Chen et al. (2006) [2], Xie et al. (2006) [4], Hettiarachchi et al. (2007, 2009) [5,6], Durmusoglu et al. (2006) [7], Xue et al. (2007) [8].

Nastev et al. (2006) established a coupled model to study the water flow, multi-component gas transport and heat conduction in MSW landfills. A software TOUGH2-LGM was developed to couple analysis of landfill gas transport and heat conduction. Based on porous media fluid dynamics, Chen et al. (2006) and Xie et al. (2006) proposed a solid-liquid-gas coupled model by considering the deformation, seepage and degradation in MSW landfills. Using finite difference method, the model was applied to simulate settlement and gas pressure change during filling phase and after the closure of a landfill. Hettiarachchi et al. $(2007,2009)$ presented a one-dimensional model could predict settlement at variable 
moisture and pressure conditions in MSW landfills. Settlement, gas pressure, moisture content and waste density were analyzed for a hypothetical MSW landfill. Durmusoglu et al. (2006) proposed a one-dimensional solid-liquid-gas coupled model to study solid-liquid-gas interactions saturated and unsaturated landfills respectively. Xue et al. (2007) established a two-dimensional model for the analysis of solid-liquid-gas interactions in landfills. Gas pressure, liquid saturation, settlement and porosity were analyzed for a hypothetical landfill cell with a gas collection well by using the model.

In this manuscript, a biochemo-hydro-mechanical (BCHM) coupled model is presented based on unsaturated seepage theory, anaerobic degradation model of MSW, and stress-degradation compression model of MSW skeleton. Finite element method is used to numerically solve the BCHM model. The functionality of the numerical formulation was examined by using a hypothetical landfill sample with high kitchen waste, which represents classic component characteristic of MSW in Chinese landfills. Variations of saturation, gas pressure, and settlement were analyzed in this paper.

\section{BCHM Coupled Model}

\section{One-stage Anaerobic Degradation Model of MSW}

The process of MSW degradation is very complicated, which is generally can be divided into two main stages: aerobic stage and anaerobic stage. MSW will undergo aerobic biodegradation immediately after the deposition in a landfill. However, as more wastes are added and particularly if the landfill is subsequently capped, overlying layers impede airflow and the dominant biochemical reactions become anaerobic. The anaerobic degradation process is a major process of MSW degradation [7,8,9,10]. Degradable component in MSW is mainly of cellulose [11], substrate of anaerobic is simplified to be cellulose hydrate by considering that the degradable component of MSW contains a large amount of intra-particle water. The anaerobic digestion of cellulose hydrate can be expressed as follow:

$$
\mathrm{C}_{6} \mathrm{H}_{10} \mathrm{O}_{5}\left(\cdot \alpha \mathrm{H}_{2} \mathrm{O}\right)+\mathrm{H}_{2} \mathrm{O} \rightarrow 3 \mathrm{CO}_{2}+3 \mathrm{CH}_{4}+(\alpha-1) \mathrm{H}_{2} \mathrm{O}
$$

Where: $\mathrm{C}_{6} \mathrm{H}_{10} \mathrm{O}_{5}\left(\cdot \alpha \mathrm{H}_{2} \mathrm{O}\right)$ is degradable component of MSW with intra-particle water; $\alpha$ is the ratio coefficient of intra-particle water mass.

First-order kinetics of the substrate degradation has been successfully used for the description of the anaerobic degradation [4]. Biodegradation model for solid mass loss is expressed as follow:

$$
m_{s d}(t)=\left(1-e^{-k t}\right) m_{s d 0}
$$

Where: $m_{s d 0}$ is initial mass of degradable component; $k$ is rate constant of biodegradation reaction.

Degradable component in MSW can be classified as solid phase (cellulose) and liquid phase (intra-particle water). During biodegradation process, solid phase is transformed to landfill gas (carbon dioxide and methane gas mixture), and intra-particle water is released to pore space at the same time. The water consumption due to anaerobic reaction is assumed to be provided by intra-particle water. According to the conservation of mass, the source terms of water release and landfill gas generation due to degradation are expressed as:

$$
\begin{aligned}
& f_{w}=\frac{18(\alpha-1)}{162+18 \alpha} k e^{-k t} \mathrm{~d} m_{s d 0} \\
& f_{g}=\frac{180}{162+18 \alpha} k e^{-k t} \mathrm{~d} m_{s d 0}
\end{aligned}
$$

Where: $f_{w}, f_{g}$ are source terms of water release and landfill gas generation respectively; $\mathrm{d} m_{s d 0}$ is degradation component mass per volume in MSW. 


\section{Liquid Flow and Gas Transportation in MSW}

Liquid flow and gas transportation in MSW models are based on porous media fluid dynamics [12]. In addition to the basic assumptions for continuous porous media, a few assumptions are applied to derive the governing equations. Liquid is assumed to be uncompressible, however gas is compressible and ideal gas law is satisfied. Velocities of liquid and gas are calculated by using Darcy's law. The effect of degradation on porosity is assumed to be neglected.

Based on above assumptions, the mass conservation equations for liquid phase and gas phase in MSW can be expressed as follow:

$$
\begin{aligned}
& \rho_{\mathrm{w}} \frac{\partial}{\partial t}(n S)=\rho_{\mathrm{w}} \nabla \cdot\left[\mathbf{k}_{\mathrm{w}} \nabla \cdot\left(u_{w}+\rho_{w} g y\right)\right]+f_{w} \\
& \frac{\partial}{\partial t}\left[\rho_{g} n(1-S)\right]=\nabla \cdot\left[\mathbf{k}_{\mathrm{g}} \nabla \cdot\left(\rho_{g} u_{g}\right)\right]+f_{\mathrm{g}}
\end{aligned}
$$

Where: $n$ is porosity; $S$ is liquid saturation; $\rho_{\mathrm{w}}, \rho_{g}$ are density of liquid and gas respectively; partial differential operator $\nabla .=\frac{\partial}{\partial x}+\frac{\partial}{\partial y}+\frac{\partial}{\partial z}: \mathbf{k}_{\mathbf{w}}, \mathbf{k}_{\mathbf{g}}$ are effective permeability coefficient matrix for liquid and gas respectively, which can be expressed as:

$$
\mathbf{k}_{\mathrm{w}, \mathrm{g}}=\left[\begin{array}{ccc}
1 & 0 & 0 \\
0 & 1 & 0 \\
0 & 0 & 1
\end{array}\right] \frac{k_{\mathrm{s}} k_{r w, g}}{\mu_{\mathrm{w}, \mathrm{g}}}=\left[\begin{array}{ccc}
1 & 0 & 0 \\
0 & 1 & 0 \\
0 & 0 & 1
\end{array}\right] \frac{k_{0} n^{3}}{(1-n)^{2}} \frac{k_{r w, g}}{\mu_{\mathrm{w}, \mathrm{g}}}
$$

Where $k_{\mathrm{s}}$ is intrinsic permeability; $k_{0}$ is initial intrinsic permeability; $\mu_{\mathrm{w}, \mathrm{g}}$ is coefficient of viscosity for liquid and gas respectively; $k_{r w, g}$ is relative permeability for liquid and gas respectively, which can be described by van-Genuchten model [13].

Liquid saturation of MSW can be expressed by a function of matrix suction under isothermal condition, the van-Geunchten model is used to describe the relationship between saturation and matrix suction in this paper.

The mass conservation equation for liquid phase (Eq.5) and gas phase (Eq.6) can be further expressed as:

$$
\begin{aligned}
& \rho_{\mathrm{w}} n \frac{\partial S}{\partial s}\left(\frac{\partial u_{g}}{\partial t}-\frac{\partial u_{w}}{\partial t}\right)=\rho_{\mathrm{w}} \nabla \cdot\left[\mathbf{k}_{\mathbf{w}} \nabla \cdot\left(u_{w}+\rho_{w} g y\right)\right]+f_{w}-\rho_{\mathrm{w}} S \frac{\partial}{\partial t}\left(\frac{\mathrm{d} w}{\mathrm{~d} z}\right) \\
& \rho_{g} n \frac{\partial S}{\partial s} \frac{\partial u_{w}}{\partial t}+\left[\frac{n(1-S) M}{R T}-\rho_{g} n \frac{\partial S}{\partial s}\right] \frac{\partial u_{g}}{\partial t}=\nabla \cdot\left[\mathbf{k}_{\mathbf{g}} \nabla \cdot\left(\rho_{g} u_{g}\right)\right]+f_{g}-\rho_{g}(1-S) \frac{\partial}{\partial t}\left(\frac{\mathrm{d} w}{\mathrm{~d} z}\right)
\end{aligned}
$$

Where: $u_{w}$ is pore water pressure; $u_{g}$ is pore gas pressure; $w$ is the vertical displacement of MSW.

\section{Compression of MSW Skeleton}

The deformation of MSW skeleton is assumed to be occurred only in vertical. Stress equilibrium equation of MSW skeleton can be expressed as:

$$
\frac{\mathrm{d} \sigma_{z}}{\mathrm{~d} z}+\mathrm{d} b_{z}=0
$$


Where: $b_{z}$ is body force; $\sigma_{z}$ is total stress, which can be calculated according to the effective principle of unsaturated soil proposed by Bishop and Blight [14]:

$$
\sigma_{z}=\sigma^{\prime}+u_{g}-\chi\left(u_{g}-u_{w}\right)
$$

Where: $\sigma^{\prime}$ is vertical effective stress; $\chi$ is parameter related to saturation, which is assumed to be equal to saturation in this paper.

The compression characteristics of MSW is of greater complexity than soils, whose solid skeleton can be normally regarded as inert materials. Based on laboratory tests on borehole samples and artificial wastes, a one-dimensional compression model which considers the coupled effect of stress and age is proposed to express mechanical compression of MSW [15]:

$$
\frac{\mathrm{dw}}{\mathrm{dz}}=\frac{C_{C}^{\prime}}{\ln 10 \cdot \sigma^{\prime}} \mathrm{d} \sigma^{\prime}+\left[\varepsilon_{b c U}+\left(C_{C \infty}^{\prime}-C_{C}^{\prime}\right) \log \frac{\sigma^{\prime}}{\sigma_{0}}\right] c e^{-c t} \mathrm{~d} t
$$

Where: $C_{C}^{\prime}$ is modified primary compression index for fresh MSW; $C_{C \infty}^{\prime}$ is modified primary compression index for decomposed MSW; $\sigma_{0}$ is the pre-consolidation stress for MSW; $\varepsilon_{b c U}$ is the sum of ultimate volumetric strains of decomposition compression and mechanical creep under pre-consolidation stress $\sigma_{0} ; c$ is the decomposition compression rate constant related to degradation process.

According to Eq. 11 and 12, Eq.10 can be further expressed as:

$$
\frac{\mathrm{d}}{\mathrm{d} z}\left(D_{1}\left(\sigma^{\prime}\right) \frac{\mathrm{d} w}{\mathrm{~d} z}\right)-\frac{\mathrm{d}}{\mathrm{d} z}\left[D_{1}\left(\sigma^{\prime}\right) D_{2}\left(\sigma^{\prime} t\right)\right] \mathrm{d} t+(1-S) \frac{\mathrm{d} u_{g}}{\mathrm{~d} z}+S \frac{\mathrm{d} u_{w}}{\mathrm{~d} z}+\mathrm{d} b_{z}=0
$$

Where:

$$
\begin{aligned}
& D_{1}\left(\sigma^{\prime}\right)=\ln 10 \cdot \sigma^{\prime} / C_{C}^{\prime}, \\
& D_{2}\left(\sigma^{\prime}, t\right)=\left[\varepsilon_{b c U}+\left(C_{C \infty}^{\prime}-C_{C}^{\prime}\right) \log \frac{\sigma^{\prime}}{\sigma_{0}}\right] c e^{-c t} .
\end{aligned}
$$

The BCHM coupled model is constituted by Eq. 8, 9 and 13. Solving variables of model includes pore water pressure, pore pressure and compression displacement of MSW. The BCHM model is solved by using finite element method due to its highly nonlinear.

\section{Analysis of Interactions in Landfills with High Kitchen Waste Content}

Different from developed countries, MSW in China contains a large amount of kitchen waste content. The degradation rate of MSW with high kitchen waste content is very high at preliminary stage, and interaction of degradation-compression-seepage is obvious. A hypothetical MSW landfill cell with a thickness of $5 \mathrm{~m}$ is designed to investigate the complex interaction in MSW landfills with high kitchen waste content. As shown in Fig. 1, the MSWs are assumed to have the same age, the top boundary is impervious to liquid but free for gas flow, and the bottom boundary is impervious to both liquid and gas to model a failed drainage, which is common in Chinese landfills. The changes in the saturation, pore gas pressure and settlement are simulated by using BCHM coupled model. MSW parameters are determined based on the results of other researchers $[8,11,16]$. The obtained results are shown in Fig. 2-4.

As shown in Fig. 2, a large amount of leachate is generated in MSW within a short period of time. The average saturation increases from $40 \%$ (initially) to $88 \%$, and the leachate mound height (the region with saturation $>90 \%$ ) reaches $2.5 \mathrm{~m}$. The change in saturation in MSW is significant. High water content also leads to lower gas permeability, which causes a significant increase in the pore gas pressure (see Fig. 3). The pore gas pressure at the bottom increases to $40.9 \mathrm{kPa}$. A loss of degradable components in MSW can 
weaken the solid skeleton significantly. Settlement strain after dissipation of pore gas pressure is approximately $55 \%$, and total settlement reached $1.43 \mathrm{~m}$ in 1 year after disposed (see Fig. 4).

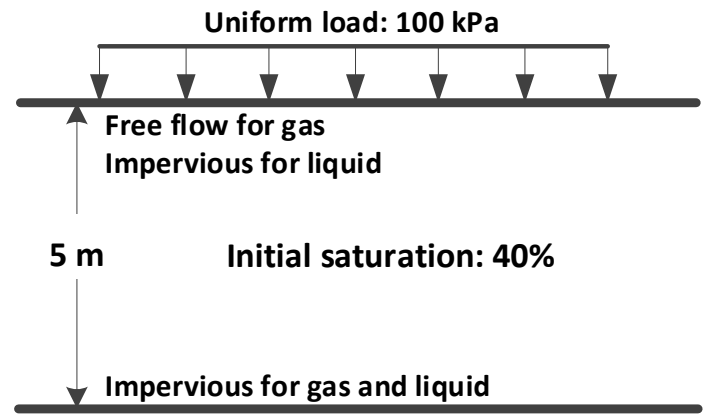

Fig.1 Case of MSWs with High Kitchen Contents

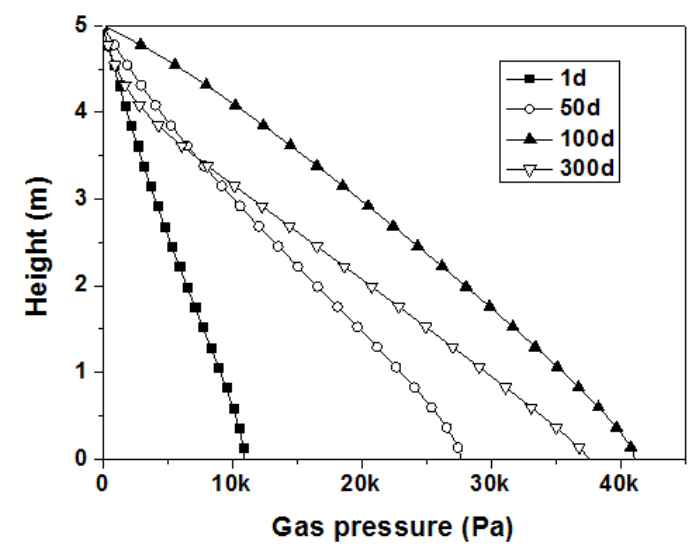

Fig. 3 Pore Gas Pressure Variation in Vertical

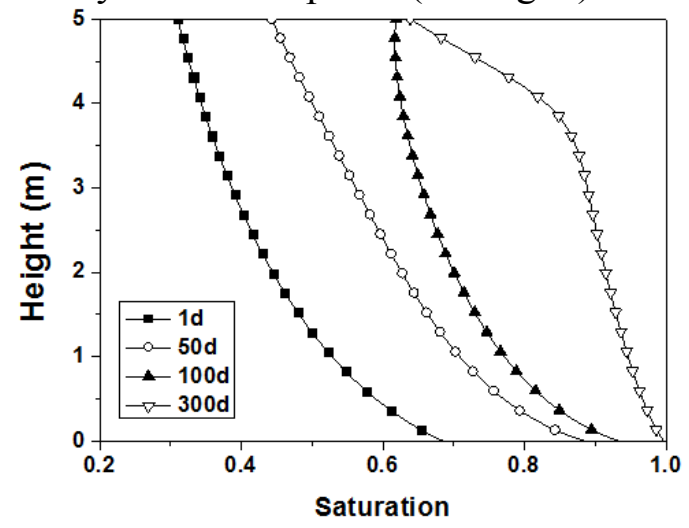

Fig. 2 Distribution of Saturation

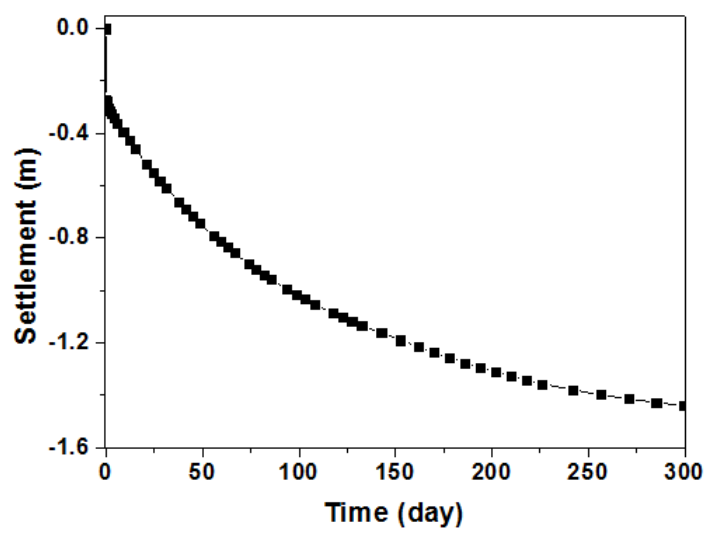

Fig. 4 Settlement of MSW Varying with Time

The obtained results above indicate that the leachate mound tends to be high in landfills with high kitchen waste content due to a large amount of intra-particle water released during degradation. Transportation of landfill gas is impeded by high leachate mound. Rapid degradation at early stage can results in a large post-closure settlement.

\section{Conclusions}

1. There are strong interactions of degradation- compression-seepage in MSW. A BCHM coupled model is proposed by study these complex interactions by considering the change of engineering properties due to degradation, such as leachate and gas generation, weaken of solid skeleton, and permeability decease.

2. High water content in MSW is an important characteristic of landfills with high kitchen waste content because a large amount of intra-particle water is released during degradation. This release leads to a high leachate mound and to gas flow impediment, which may induce landfill slope failure and low landfill gas collection efficiency, respectively.

\section{Acknowledgements}

The financial supports received from the National Basic Research Program of China (973 Program) via Grant No. 2012CB719806 is gratefully acknowledged.

\section{References}

[1] The Statistical Yearbook of Chinese Urban Construction 2007. China Architecture \& Building Press. 
[2] Y.M. Chen, Y. Xie, L.T. Zhan. One-dimensional consolidation model for landfills considering solid-liquid-gas interaction J. Chinese Journal of Geotechnical Engineering, 2006, 28(2): 184-190.

[3] M. Nastev, R. Therrien, R. Lefebvre, P. Gelinas. Gas production and migration in landfills and geological materials J. Journal of contaminant hydrology, 2001, 52: 187-211.

[4] Y. Xie, Y.M. Chen, X.W. Tang, D.S. Ling, H. Ke. Mathematical model for landfill settlement considering gas-solid coupling effect J. Chinese Journal of Rock Mechanics and Engineering, 2006, 25(3): 601-608.

[5] C.H. Hettiarachchi, J.N. Meegoda, J. Tavantzis, P. Hettiaratchi. Numerical model to predict settlements coupled with landfill gas pressure in bioreactor landfills J. Journal of Hazardous Materials, 2007, B139: 514-522.

[6] C.H. Hettiarachchi, J.N. Meegoda, P. Hettiaratchi. Effects of gas and moisture on modeling of bioreactor landfill settlement J. Waste Management, 2009, 29: 1018-1025.

[7] E. Durmusoglu, M.Y. Corapciglu, K. Tuncay. Modelling of settlement in saturated and unsaturated municipal landfills [J]. International Journal of Geomechanics, 2006, 6(4): 269-278.

[8] Q. Xue, L. Liu, B. Liang, Y. Zhao, Y.B. Wang. A gas-hydraulic-solid coupling dynamics model under landfill settlement J. Chinese Journal of Rock Mechanics and Engineering, 2007, 26: 3473-3478.

[9] G.J. Farquhar and F.A. Rovers. Gas production during refuse decomposition. Waste, air and soil pollution .v.2 M. 1973:493-495.

[10] Parker. Behavior of wastes in landfill Leachate. Practice waste management. England, Chapter 7 M.1983: 205-217.

[11] J.R. McDougall. A hydro-bio-mechanical model for settlement and other behaviour in landfilled waste J. Computers and Geotechnics. 2007, 34: 229-246.

[12] J.C. Parker. Multiphase flow and transport in porous media. Reviews of Geophysics M. 1989, 27(3): 311-328.

[13] C. Zeiss and M. Uguccioni. Mechanisms and patterns of leachate flow in municipal Solid Waste Landfills J. Journal of environmental systems. 1994-95, 23(3), 247-270.

[14] A.W. Bishop, G.E. Blight. Some aspects of effective stress in saturated and partly saturated soils [J]. Geotechnique. 1963, 13(3): 177-197

[15] Y.M. Chen, H. Ke, D.G. Fredlund, et al. Secondary compression of municipal solid wastes and a compression model for predicting settlement of municipal solid waste landfills J. Journal of geotechnical and geoenvironmental engineering, 2010, 136(5): 706-717.

[16] Y.C. Li, H.L. Liu, P.J. Cleall, et al. Influences of operational practices on municipal solid waste landfill storage capacity J. Waste Management \& Research, 2013, 31(3): 273-282. 